\title{
Predictive formula of cervical lordosis in asymptomatic young population
}

\author{
Yuchen Zhu ${ }^{1,2 \dagger}$, Zhongcheng An ${ }^{1 \dagger}$, Yingjian Zhang ${ }^{1,2}, \mathrm{Hao} \mathrm{Wei}^{1}$ and Liqiang Dong ${ }^{1 *}$
}

\begin{abstract}
Background: Not a large number of previous studies have reported the normal sagittal balance of the cervical spine and physiological cervical lordosis (CL) has not been clearly defined yet.

Methods: This was a prospective radiological analysis of asymptomatic subjects. The following cervical sagittal parameters were measured: $\mathrm{CL}$, thoracic inlet angle (TIA), T1 slope (T1S), neck tilt (NT), and C2-7 sagittal vertical axis (C2-7 SVA). The Pearson correlation test was calculated, and the stepwise multiple regression analysis was conducted by using the $\mathrm{CL}$ (dependent variable) and the other cervical sagittal parameters (independent variables) to determine the best sets of predictors. A paired sample t test was conducted between the predicted and measured values.

Results: The mean age of 307 participants was $24.54+3.07$. The mean CL, TIA, T1S, NT, and C2-C7 SVA was $17.11^{\circ} \pm$ $6.31^{\circ}, 67.87^{\circ} \pm 7.78^{\circ}, 25.84^{\circ} \pm 5.36^{\circ}, 42.53^{\circ} \pm 6.68^{\circ}$, and $14.60 \pm 8.20 \mathrm{~mm}$, respectively. The formula was established as follows: $\mathrm{CL}=0.762 \times \mathrm{T} 1 \mathrm{~S}-0.392 \times \mathrm{C} 2-\mathrm{C} 7 \mathrm{SVA}+0.25 \times \mathrm{TIA}-13.795\left(R=0.812, R^{2}=0.660\right)$ (stepwise multiple regression) and $\mathrm{CL}=0.417 \times \mathrm{TIA}-11.193\left(R=0.514, R^{2}=0.264\right)$ (simple linear regression). There was no statistical difference between the predicted $C L$ and the measured $C L(t=0.034, P=0.973)$.

Conclusions: There was a significant correlation between $C L$ and other cervical sagittal parameters, including TIA, T1S, NT, and C2-C7 SVA in asymptomatic Chinese population. The results of this study may serve as a normal reference value for the study of asymptomatic population.
\end{abstract}

Keywords: Cervical sagittal alignment, Cervical lordosis, Thoracic inlet angle, Regression analysis, Predictive formula

\section{Background}

The spine has a certain physiological curvature in the sagittal plane. For the human body always tends to obtain a stable posture at the minimum energy expenditure when standing and walking, it is critical to maintaining the sagittal balance of the spine [1]. The cervical spine, as the most mobile part relative to the rest of the spinal column and also supports the mass of the head, plays a pivotal role in sagittal spinal balance. Any deviations from the normal alignment of the mass of the head would result in a biomechanical imbalance of the cervical spine and an increase in muscular energy expenditure, and bringing a variety of disorders and complications. With the deepening of research, sagittal plane alignment is increasingly recognized as a critical parameter in the setting of adult

\footnotetext{
*Correspondence: dlq58@126.com

${ }^{\dagger}$ Yuchen Zhu and Zhongcheng An contributed equally to this work. 'Department of Spine Surgery, Second Affiliated Hospital of Zhejiang Chinese Medical University, Hangzhou 310005, People's Republic of China Full list of author information is available at the end of the article
}

spinal deformity [2-4]. It has become evident that good clinical outcomes in the treatment of spinal deformity require proper alignment [5]. In recent years, significant progress has been made in the study of the global spinal sagittal alignment parameters. However, the research is largely focused on the spine-pelvic region. Comparatively, the cervical sagittal parameters that affect clinical outcomes of various cervical diseases have not been well defined yet. In addition, there is no set standard to address the amount of correction to be achieved in cervical deformity correction surgery.

However, proper diagnostic evaluation and optimal treatment approaches for spinal deformity need to be based on the investigation of healthy individuals [5]. Herein, we analyzed the cervical sagittal parameters of asymptomatic subjects within a certain age range. The results of this study may serve as a normal reference value of ideal CL for the evaluation of sagittal balance or planning of a fusion angle in the cervical spine. 


\section{Methods}

\section{Study population}

From March 2019 to June 2019, the imaging data of volunteers who underwent cervical spine health checkup in our hospital were collected. The study protocol was approved by the Institutional Review Board, and informed consent was obtained from all participants.

The inclusion criteria were as follows: (1) The age was 18-30 years old, (2) no history of symptoms, diagnosis, and treatment related to the whole spine, hip joint, and lower extremity. No history of chronic pain in the neck or shoulder, no history of spinal diseases or surgery, no history of pelvis, hip joint, or lower extremity diseases; (3) no cervical instability and spondylolisthesis showed on X-ray; (4) no significant scoliosis in the coronal plane (Cobb angle $<10^{\circ}$ ), and no kyphosis deformity in the sagittal plane. A total of 307 volunteers were enrolled, including 144 males and 163 females.

\section{Radiographic measurement}

All the participants had undergone standard anterior and lateral X-rays of the cervical spine. The cervical spine parameters were measured independently using hospital image archiving and communication system (Centricity RIS/PACS, GE healthcare) by two authors. And each result was averaged.

The parameters are as follows (Figs. 1 and 2, measurements of the parameters).

(1) The thoracic inlet angle (TIA): an angle formed by a line from the center of the T1 upper endplate (T1UEP) vertical to the T1UEP and a line connecting the center of the T1UEP and the upper end of the sternum. (2) The T1 slope (T1S): an angle formed between the horizontal plane and the T1UEP. (3) The neck tilt (NT): an angle formed by a line drawn in the upper end of the sternum and a line connecting the center of the T1UEP and the upper end of the sternum. (4) The cervical lordosis of $\mathrm{C} 2-7$ (CL): a Cobb angle between the $\mathrm{C} 2$ lower endplate and the $\mathrm{C} 7$ lower endplate. (5) The $\mathrm{C} 2-7$ sagittal vertical axis (C2-7 SVA): the distance between the $\mathrm{C} 2$ plumb line and the posterior $\mathrm{C} 7$ upper endplate.

\section{Statistical analysis}

The statistical analyses were performed using SPSS version 20.0 (IBM Corporation, Armonk, NY, USA). There

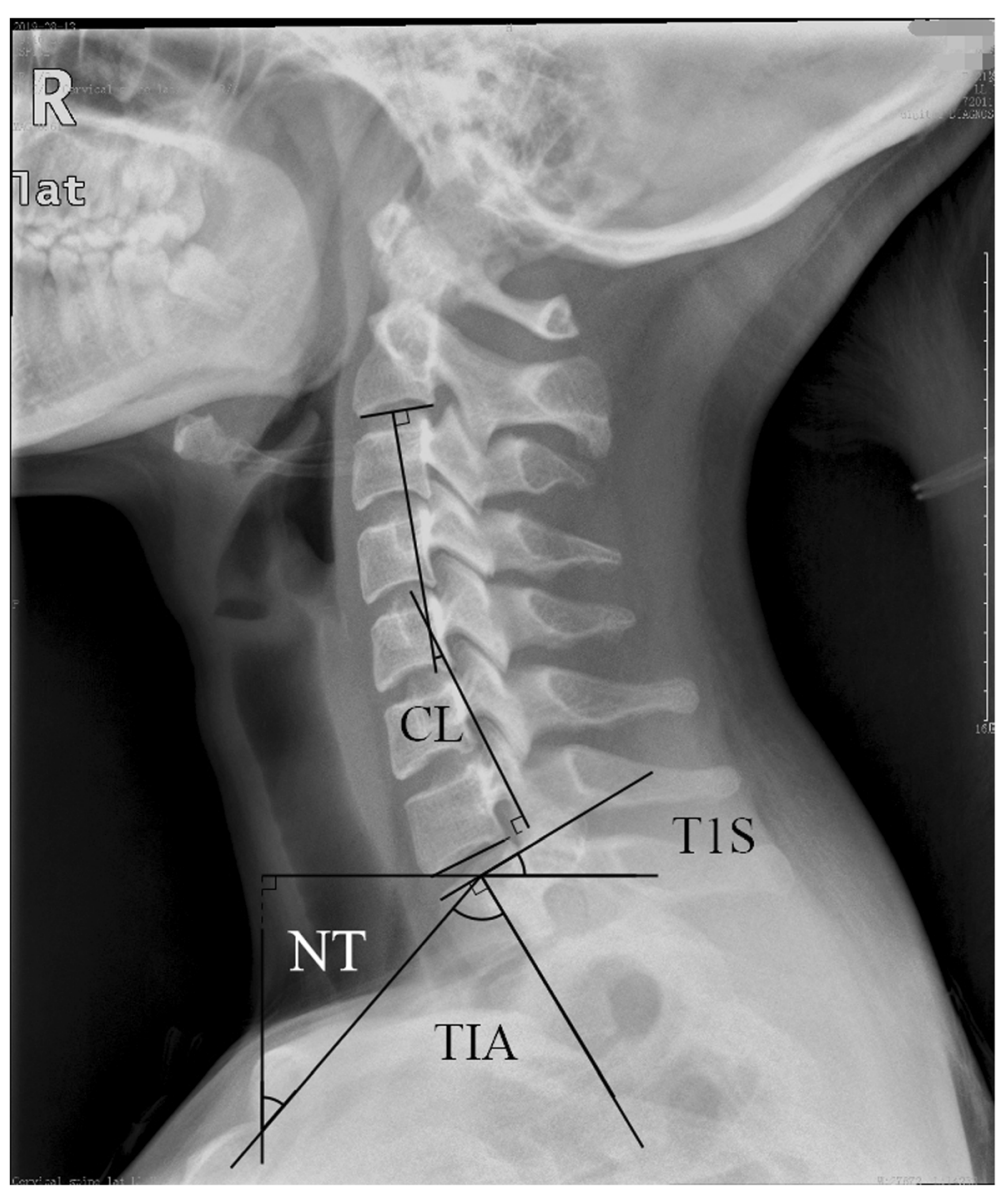

Fig. 1 Measurements of the parameters 


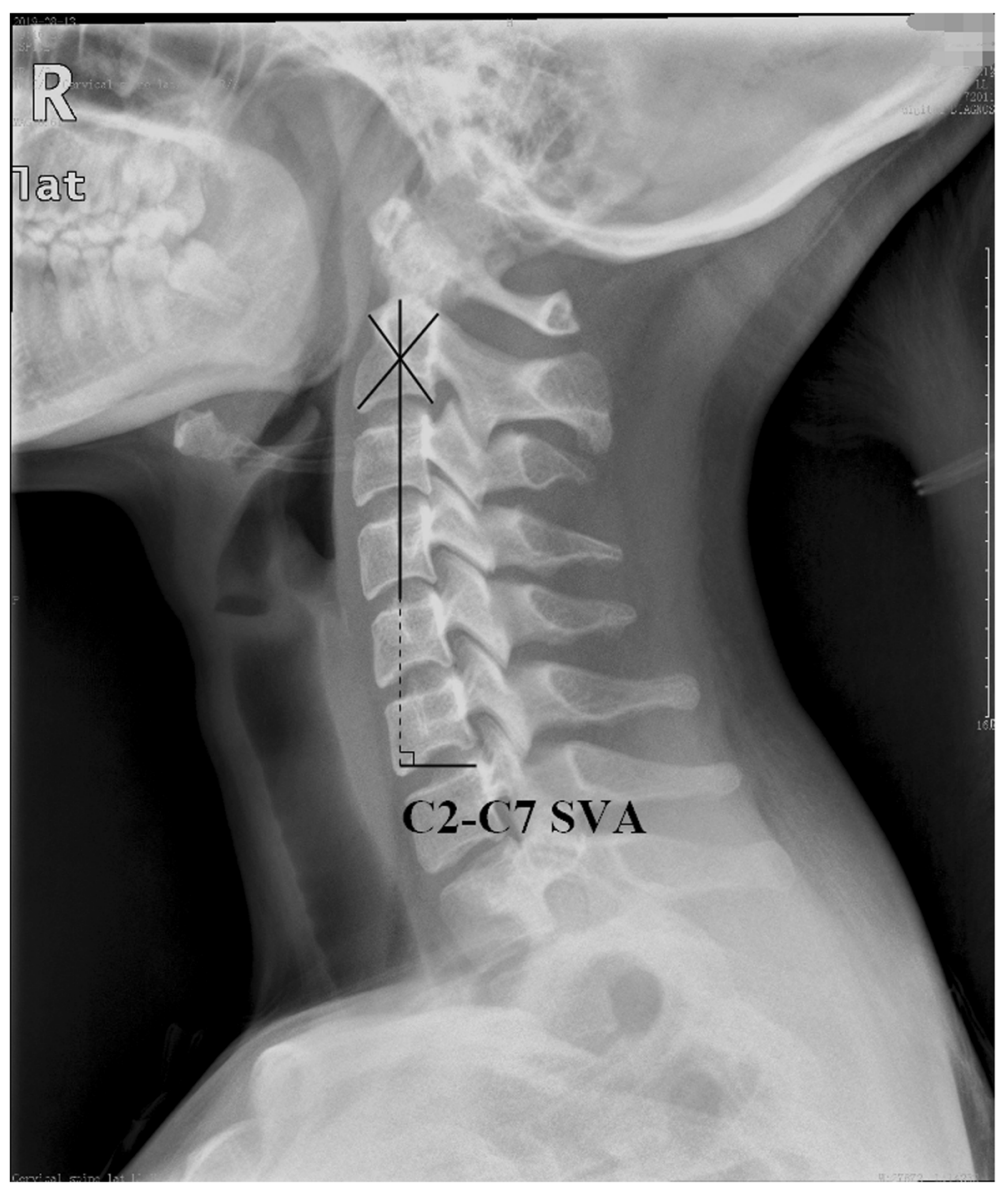

Fig. 2 Measurements of the parameters

were three steps: (1) descriptive statistics analysis of demographic data and parameters; (2) correlation analysis between CL and other parameters using the Pearson correlation coefficient; (3) stepwise multiple regression and simple regression analysis of parameters, and $\mathrm{CL}$ was the dependent variable. A $P$ value of $<0.05$ was considered statistically significant.

\section{Results}

\section{Demographic data and sagittal parameters}

The mean age of participants was $24.54+3.07$ (range, 18 to 30 years old). The average value, standard deviation, range, and standard error of CL, TIA, T1S, NT, and C2C7 SVA were shown in Table 1. The mean CL of 307 participants was $17.11 \pm 6.31^{\circ}, 17.97 \pm 6.30^{\circ}$ in males and $16.35 \pm 6.23^{\circ}$ in females. The mean CL in males was larger than that in females $(t=-2.246, P=0.025)$. The mean TIA was $67.87 \pm 7.78^{\circ}, 70.33 \pm 9.21^{\circ}$ in males, and $65.69 \pm 5.40^{\circ}$ in females. The TIA in males was significantly higher than that in females $(t=-5.457, P=0.000)$. The results of Pearson correlation analysis between CL and other parameters were shown in Table 2. CL was significantly correlated with T1S, C2-C7 SVA, and TIA $(P<0.05)$, while $\mathrm{CL}$ was not correlated with NT $(P=$ $0.762)$.

\section{Predictive formula of cervical lordosis}

A predictive formula with relevant variables, including TIA, T1S, C2-C7 SVA (NT was excluded during the regression analysis), for CL was established using stepwise multiple regression analysis of the above parameters (Tables 3 and 4). That was CL $=0.762 \times \mathrm{T} 1 \mathrm{~S}-0.392 \times \mathrm{C} 2-$ C7 SVA + $0.25 \times$ TIA $-13.795\left(R=0.812, R^{2}=0.660\right)$.

Table 1 Average value, standard deviation, range, and standard error

\begin{tabular}{llll}
\hline Parameters & Average value $(\bar{x} \pm s)$ & Range & Standard error \\
\hline Age & $24.54 \pm 3.07$ & $18-30$ & 0.173 \\
CL (degrees) & $17.11 \pm 6.31$ & $-8.94-28.44$ & 0.360 \\
TIA (degrees) & $67.87 \pm 7.78$ & $43.02-88.98$ & 0.444 \\
T1S (degrees) & $25.84 \pm 5.36$ & $12.00-41.20$ & 0.306 \\
NT (degrees) & $42.53 \pm 6.68$ & $27.88-59.12$ & 0.381 \\
C2-C7 SVA (mm) & $14.60 \pm 8.20$ & $-6.00-40.00$ & 0.468 \\
\hline
\end{tabular}


Table 2 Pearson correlation analysis

\begin{tabular}{lll}
\hline & $\mathrm{CL}$ & $P$ value \\
\hline TIA & 0.514 & 0.000 \\
T1S & 0.620 & 0.000 \\
NT & 0.092 & 0.109 \\
C2-C7 SVA & -0.196 & 0.001 \\
\hline
\end{tabular}

In the formula above, T1S and C2-C7 SVA were orientation parameters influenced by the posture. While TIA was a constant morphological parameter, not influenced by the posture and unchanged in adulthood. Thus, a simplified formula for CL was obtained from simple linear regression analysis with TIA. That was $\mathrm{CL}=0.417 \times$ TIA $-11.193 \quad\left(R=0.514, \quad R^{2}=0.264\right)$ (Table 4). Based on the TIA values measured, the predictive value of $\mathrm{CL}$ was calculated to be $17.10 \pm 3.24^{\circ}$. There was no statistical difference between the predicted value and the measured value using the paired sample $t$ test $(t=0.034, P=0.973)$.

\section{Discussion}

The spinal regions (the pelvis and the lumbar, thoracic, and cervical regions) are not independent of one another, and they have multiple significant correlations. As described by Dubousset in his "Conus of Economy" theory, the body adapts to changes in balance in order to regulate the center of gravity over as narrow a perimeter as possible [6]. Sagittal alignment has important implications for muscular energy expenditure in the maintenance of posture. A cervical deformity can lead to compensatory mechanisms such as knee flexion, pelvic retroversion, thoracic hypokyphosis, and lumbar hyperlordosis, to maintain a balanced, upright posture and horizontal gaze $[7,8]$. And in cervical spine sagittal parameters, T1S, C2-C7 SVA, chin-brow to vertical angle (CBVA) increases while NT decreases as compensatory mechanisms. Indeed, physiological cervical lordosis is an essential condition for spinal coupling motion. The morphological changes of the cervical sagittal plane will eventually lead to changes in the segment subjected to the greatest stress during the motion and accelerate its degeneration.

The primary purpose of cervical deformity correction surgery is to maintain or restore the horizontal gaze, decompress the spinal cord or nerve root, and reconstruct the cervical spine alignment. There currently exist no

Table 3 Stepwise multiple regression analysis

\begin{tabular}{lllll}
\hline Step & $R$ & $R^{2}$ & Adjusted $R^{2}$ & independent variables \\
\hline 1 & 0.620 & 0.384 & 0.382 & $\mathrm{~T}_{1} \mathrm{~S}$ \\
2 & 0.769 & 0.691 & 0.588 & $\mathrm{~T}_{1} \mathrm{~S}, \mathrm{C}_{2}-\mathrm{C}_{7}$ SVA \\
3 & 0.812 & 0.660 & 0.657 & $\mathrm{~T}_{1} \mathrm{~S}, \mathrm{C}_{2}-\mathrm{C}_{7}$ SVA, TIA \\
\hline
\end{tabular}

commonly acknowledged criteria for cervical sagittal plane correction, but to reconstruct and maintain the global spinal sagittal alignment as far as possible has become a consensus $[9,10]$. In recent years, many studies have concentrated on the correlation of cervical alignment parameters to disability scores and myelopathy outcomes. Tang et al. noted that the $\mathrm{C} 1-\mathrm{C} 2$ lordosis angle is an essential parameter for regulating the angle of gaze in cervical reconstructive surgery, and the postoperative $\mathrm{C} 2-\mathrm{C} 7$ sagittal vertical axis (SVA) is significantly correlated with health-related quality-of-life (HRQOL) scores [11]. Ames et al. also suggested correlations between radiographical parameters in the cervical spine and HRQOL outcomes [12]. The authors believed that the TIA and T1S might be used as parameters to evaluate sagittal balance, predict physiological alignment, and guide deformity correction of the cervical spine. According to the formula, geometrically, $\mathrm{TIA}=\mathrm{T} 1 \mathrm{~S}+\mathrm{NT}$, it could be presumed that large TIA increases T1S and finally increase CL to obtain a horizontal gaze and sagittal alignment of the cervical spine with minimum energy expenditure, and vice versa. On the basis of previous studies, we attempt to establish a further relationship between the alignment parameters, that is, a predicting formula.

However, whether spinopelvic parameters or cervical spine parameters, differences exist in population from different regions. The results of the cervical spine parameters of 77 asymptomatic Korean adult volunteers measured by Lee et al. showed that the mean TIA, T1S, and NT were $69.5^{\circ}, 25.7^{\circ}$, and $43.7^{\circ}$, respectively [13]. Zhang et al. reported that the mean TIA, T1S, and NT of 67 Chinese adults with mild neck symptoms were $72.8^{\circ}, 22.3^{\circ}$, and $49.7^{\circ}$, respectively [14]. Moreover, radiographic measurements of 120 asymptomatic American adult volunteers performed by Iyer et al. showed the mean TIA, T1S, and NT were $79.8^{\circ}, 26.1^{\circ}$, and $51^{\circ}$, respectively [15]. Thus, it seems to be significant to investigate correlations of sequential parameters and construct predictive formulas of postoperative spinal alignment of each region in order to plan surgery for cervical deformity correction optimally.

In this study, we observed the cervical sagittal parameters of 307 asymptomatic Chinese adult volunteers, and the mean CL came to $17.11 \pm 6.31^{\circ}$. The Pearson correlation coefficient and linear regression models found that CL was significantly correlated with TIA, T1S, NT, and C2-C7 SVA, which was consistent with previous studies $[1,8,12]$. Then, a predictive formula with these relevant variables was made using stepwise multiple regression analysis of the above parameters. The following formula was established, and TIA, T1S, and C2-C7 SVA were important predictive variables: $\mathrm{CL}=0.762 \times \mathrm{T} 1 \mathrm{~S}-$ $0.392 \times \mathrm{C} 2-\mathrm{C} 7 \quad \mathrm{SVA}+0.25 \times \mathrm{TIA}-13.795 \quad(R=0.812$, 
Table 4 The coefficients and constants of two predictive formulas

\begin{tabular}{llll}
\hline Model & Regression coefficients & Standardized coefficients & $P$ value \\
\hline Stepwise multiple regression analysis & & & 0.648 \\
T1S & 0.762 & -0.510 & 0.000 \\
$C_{2}-C_{7}$ SVA & -0.392 & 0.308 & 0.000 \\
TIA & 0.250 & 0 & 0.000 \\
Constant & -13.795 & & 0.000 \\
Simple linear regression analysis & & 0.514 & 0.000 \\
TIA & 0.417 & 0 & 0 \\
Constant & -11.193 & & \\
\hline
\end{tabular}

$R^{2}=0.660$ ), in which the determination coefficient declared a good linear correlation. However, this formula is based on young asymptomatic adults with normal spinal curvature in the sagittal plane. In pathological conditions, to adapt to the variations in the shape of the spine, several compensation mechanisms are implemented at the segmental, regional, and global levels. Moreover, T1S, as well as C2-C7 SVA, would be influenced, for it is not a constant parameter. Therefore, we consider that it is inefficient to use T1S and C2-C7 SVA to predict the extent of correction to be performed during surgery. In view of the fact that TIA is a constant morphological parameter not influenced by aging or posture, the formula $\mathrm{CL}=0.417 \times$ TIA $-11.193(R=$ $0.514, R^{2}=0.264$ ) could be a simple approach.

Age-related spinal degeneration is one of the crucial factors affecting sagittal spinal parameters [16]. For the purpose of analyzing the relationship between $\mathrm{CL}$ and other cervical sagittal parameters in normal individuals, we recruited healthy volunteers of the age-specific group (average age $24.54 \pm 3.07$ ) at which most degenerative changes in the spine have not obviously developed. These data can be a useful reference for the study of the asymptomatic population. However, it is also a limitation of our study that we could not see continuous sagittal changes according to all ages because of the certain age range of subjects. Indeed, most of the patients who need surgical intervention are of advanced age. So a further study involving an old age group is valuable for cervical surgery. Another limitation of our study is that we did not take global spinal parameters into consideration. It is recognized that cervical deformity correction should take on a comprehensive approach in assessing global cervical-pelvic relationships [12]. The spinal regions are not independent of one another, and CL as an adaptive spinal segment depends on the alignment of both thoracic and lumbar spine. In previous studies of sagittal spinal parameters, Schwab et al. proposed a simple approach of "lumbar lordosis $=$ pelvic incidence $\pm 9^{\circ}$ ", which has been verified good correlation with HROQL and applied in most reconstructive surgeries at the thoracic and lumbar spine $[5,17,18]$. Therefore, for the cervical spine, further prospective study of the relationship between predictive CL and postoperative HRQOL outcomes is needed to find out whether predictive CL predicted only by cervical sagittal parameters actually can be applied to surgical planning.

\section{Conclusions}

There was a significant correlation between $\mathrm{CL}$ and other cervical sagittal parameters including TIA, T1S, NT, and C2-C7 SVA in asymptomatic Chinese population. The results of this study may serve as a normal reference value for the study of asymptomatic population.

\section{Abbreviations}

C2-7 SVA: C2-7 sagittal vertical axis; CBVA: Chin-brow to vertical angle; CL: Cervical lordosis; HRQOL scores: Health-related quality-of-life scores; NT: Neck tilt; T1S: T1 slope; T1UEP: T1 upper endplate; TIA: Thoracic inlet angle

\section{Acknowledgements}

Not applicable.

Authors' contributions

LQD conceived and designed the study. YJZ, HW, and LQD collected the data. YCZ and ZCA analyzed and interpreted the data. YCZ, ZCA, and LQD wrote the paper. All authors read and approved the final manuscript.

\section{Funding}

Not applicable.

Availability of data and materials

The datasets generated and analyzed during the current study are available from the corresponding author on reasonable request.

\section{Ethics approval and consent to participate}

This study has obtained ethics approval of the ethics committee in our hospital and informed consent of all participants (ethical approval number: 2019-KL-141-01)

\section{Consent for publication}

Not applicable.

\section{Competing interests}

The authors declare that they have no competing interests.

\section{Author details}

${ }^{1}$ Department of Spine Surgery, Second Affiliated Hospital of Zhejiang

Chinese Medical University, Hangzhou 310005, People's Republic of China. 
${ }^{2}$ The Second Clinical Medical College of Zhejiang Chinese Medical University, Hangzhou, People's Republic of China.

Received: 10 October 2019 Accepted: 18 December 2019

Published online: 03 January 2020

\section{References}

1. Scheer Justin K, Tang Jessica A, Smith Justin S, et al. Cervical spine alignment, sagittal deformity, and clinical implications: a review. J Neurosurg Spine. 2013;19:141-59. https://doi.org/10.3171/2013.4.spine12838.

2. Emmanuelle $C-V$, Jean-Marc $M-T$, Jérôme $P$, et al. Sagittal spino-pelvic alignment in chronic low back pain. Eur Spine J. 2011;20(5 Supplement): 634-40. https://doi.org/10.1007/s00586-011-1931-2.

3. Labelle H, Mac-Thiong JM, Roussouly P. Spino-pelvic sagittal balance of spondylolisthesis: a review and classification. Eur Spine J. 2011;20(Suppl 5): 641-6. https://doi.org/10.1007/s00586-011-1932-1.

4. Li WS, Li G, Chen ZQ, et al. Sagittal plane analysis of the spine and pelvis in adult idiopathic scoliosis. Chin Med J. 2010;123(21):2978-82. https://doi.org/ 10.3760/cma.j.issn.0366-6999.2010.21.005.

5. Schwab F, Lafage V, Patel A, et al. Sagittal plane considerations and the pelvis in the adult patient. Spine (Phila Pa 1976). 2009;34(17):1828-33. https://doi.org/10.1097/brs.0b013e3181a13c08.

6. Dubousset J. Three-dimensional analysis of the scoliotic deformity. In: Weinstein S (ed) The pediatric spine: principles and practice. New York: Raven Press; 1994. p. 479-96.

7. Le Huec JC, Thompson W, Mohsinaly Y et al. Sagittal balance of the spine. Eur Spine J. 2019;28:1889-905. https://doi.org/10.1007/s00586-019-06083-1.

8. Weng C, Wang J, Tuchman A, et al. Influence of T1 slope on the cervical sagittal balance in degenerative cervical spine: an analysis using kinematic MRl. Spine (Phila Pa 1976). 2016;41(3):185-90. https://doi.org/10.1097/brs. 0000000000001353

9. Protopsaltis T, Bronsard N, Soroceanu A, et al. Cervical sagittal deformity develops after PJK in adult thoracolumbar deformity correction: radiographic analysis utilizing a novel global sagittal angular parameter, the CTPA. Eur Spine J. 2017;26(4):1111-20. https://doi.org/10.1007/s00586-0164653-7.

10. Steinmetz MP, Stewart TJ, Kager CD, et al. Cervical deformity correction. Neurosurgery. 2007;60(1):90-7. https://doi.org/10.1227/01.neu.0000215553. 49728.b0.

11. Tang JA, Scheer JK, Smith JS, et al. The impact of standing regional cervical sagittal alignment on outcomes in posterior cervical fusion surgery. Spine Journal. 2012;12(9):S65. https://doi.org/10.1016/j.spinee.2012.08.189.

12. Ames CP, Benjamin B, Scheer JK, et al. Cervical radiographical alignment: comprehensive assessment techniques and potential importance in cervical myelopathy. Spine. 2013;38(1):149-60. https://doi.org/10.1097/BRS 0b013e3182a7f449.

13. Lee SH, Kim KT, Seo EM, et al. The influence of thoracic inlet alignment on the craniocervical sagittal balance in asymptomatic adults. J Spinal Disord Tech. 2012;25(2):E41-7. https://doi.org/10.1097/BRS.0b013e3182a7f449.

14. Zhang KB, Zhan HR, Yu B, et al. Difference of sagittal parameters of adult cervical vertebrae measured by X-ray and CT imaging. J Third Military Med Univ (China). 2015;37(19):1997-2000. https://doi.org/10.16016/j.1000-5404. 201501195.

15. Iyer S, Lenke LG, Nemani VM, et al. Variations in occipitocervical and cervicothoracic alignment parameters based on age: a prospective study of asymptomatic volunteers using full-body radiographs[]]. Spine (Phila Pa 1976). 2016;41(23):1837-44. https://doi.org/10.1097/ BRS.0000000000001642.

16. Sasaki K, Hongo M, Miyakoshi N, et al. Evaluation of sagittal spine-pelvis-lower limb alignment in elderly women with pelvic retroversion while standing and walking using a three-dimensional musculoskeletal model. Asian Spine Journal. 2017:11(4):562-9. https://doi.org/10.4184/asj.2017.11.4.562.

17. Ha KY, Jang WH, Kim YH, et al. Clinical Relevance of the SRS-Schwab Classification for Degenerative Lumbar Scoliosis. Spine. 2016;41(5):E282. https://doi.org/10.1097/brs.00000 00000001229

18. Sun $X Y$, Zhang $X N$, Hai $Y$. Optimum pelvic incidence minus lumbar lordosis value after operation for patients with adult degenerative scoliosis. The Spine J. 2017;17(7):983-9. https://doi.org/10.1016/.spine e.2017.03.008.

\section{Publisher's Note}

Springer Nature remains neutral with regard to jurisdictional claims in published maps and institutional affiliations.

Ready to submit your research? Choose BMC and benefit from:

- fast, convenient online submission

- thorough peer review by experienced researchers in your field

- rapid publication on acceptance

- support for research data, including large and complex data types

- gold Open Access which fosters wider collaboration and increased citations

- maximum visibility for your research: over $100 \mathrm{M}$ website views per year

At BMC, research is always in progress.

Learn more biomedcentral.com/submissions 